\title{
How to Run Turing Machines on Encrypted Data
}

\author{
Shafi Goldwasser ${ }^{1}$, Yael Tauman Kalai ${ }^{2}$, Raluca Ada Popa ${ }^{1}$, \\ Vinod Vaikuntanathan ${ }^{3}$, and Nickolai Zeldovich ${ }^{1}$ \\ 1 MIT CSAIL \\ 2 Microsoft Research \\ 3 University of Toronto
}

\begin{abstract}
Cryptographic schemes for computing on encrypted data promise to be a fundamental building block of cryptography. The way one models such algorithms has a crucial effect on the efficiency and usefulness of the resulting cryptographic schemes. As of today, almost all known schemes for fully homomorphic encryption, functional encryption, and garbling schemes work by modeling algorithms as circuits rather than as Turing machines.

As a consequence of this modeling, evaluating an algorithm over encrypted data is as slow as the worst-case running time of that algorithm, a dire fact for many tasks. In addition, in settings where an evaluator needs a description of the algorithm itself in some "encoded" form, the cost of computing and communicating such encoding is as large as the worst-case running time of this algorithm.

In this work, we construct cryptographic schemes for computing Turing machines on encrypted data that avoid the worst-case problem. Specifically, we show:

- An attribute-based encryption scheme for any polynomial-time Turing machine and Random Access Machine (RAM).

- A (single-key and succinct) functional encryption scheme for any polynomialtime Turing machine.

- A reusable garbling scheme for any polynomial-time Turing machine.

These three schemes have the property that the size of a key or of a garbling for a Turing machine is very short: it depends only on the description of the Turing machine and not on its running time.

Previously, the only existing constructions of such schemes were for depth$d$ circuits, where all the parameters grow with $d$. Our constructions remove this depth $d$ restriction, have short keys, and moreover, avoid the worst-case running time.

- A variant of fully homomorphic encryption scheme for Turing machines, where one can evaluate a Turing machine $M$ on an encrypted input $x$ in time that is dependent on the running time of $M$ on input $x$ as opposed to the worstcase runtime of $M$. Previously, such a result was known only for a restricted class of Turing machines and it required an expensive preprocessing phase (with worst-case runtime); our constructions remove both restrictions.

Our results are obtained via a reduction from SNARKs (Bitanski et al) and an "extractable" variant of witness encryption, a scheme introduced by Garg et al. We prove that the new assumption is secure in the generic group model. We also point out the connection between (the variant of) witness encryption and the obfuscation of point filter functions as defined by Goldwasser and Kalai in 2005.
\end{abstract}

Keywords: Computing on encrypted data, Functional encryption, Fully homomorphic encryption, Turing machines, Input-specific running time. 


\section{Introduction}

Cryptographic schemes for computing on encrypted data promise to be a major focus of cryptographic research for years to come. We now have early constructions of fully homomorphic encryption, functional encryption, and attribute-based encryption, as well as more established constructions for garbling schemes. An important question for the practicality and usability of these schemes is how to model an algorithm that computes on encrypted data in cryptographic constructions.

Modeling algorithms as circuits instead of Turing machines has efficiency and usability disadvantages. Indeed, almost all known 1$]$ cryptographic constructions of fully homomorphic encryption, attribute-based encryption, functional encryption and garbling schemes for general algorithms model these algorithms as Boolean or arithmetic circuits. As a consequence, these constructions suffer from the following two disadvantages.

The first disadvantage is that evaluating an algorithm $A$ modeled as a circuit on encrypted data is at least as slow as the worst-case running time of algorithm $A$ on all inputs of a certain size. Ideally, the runtime of $A$ on input $x$ should be the time $A$ takes to run on $x$. The reason for this slowdown is that all the known transformations from Turing machines to circuits essentially work by unrolling loops to their worst-case runtime, and by considering all branches of a computation. Even if the cryptographic overhead of these schemes were zero, such worst-case runtime can still make the computation prohibitively slow: for example, the simplex algorithm for linear programming runs in polynomial time on most instances one encounters in practice, but in exponential time on rare inputs.

The second disadvantage arises for schemes that require an evaluator to obtain an encoded description of an algorithm $A$ (called a token) in order to run $A$ on the encrypted data. For example, in functional encryption, the token is a key for the algorithm $A$ and in garbling schemes, the token is the garbling of the algorithm. In these settings, modeling algorithms as circuits makes the size of the token as large as the running time of the algorithm, instead of having the token size depend only on the description of the algorithm, which can be much shorter.

The earliest example of using circuits for computing on encrypted data is Yao's secure function evaluation protocol [Yao86] which takes as input any polynomialtime computable function $f$ - specified by a circuit - and outputs a "garbled circuit" with the same input-output functionality. Such worst-case runtime also affects known two-party and multi-party protocols for general secure function evaluation [Yao86 GMW87|BGW88 CCD88].

More recent constructions for computing on encrypted data also use circuits to model computation and thus suffer from the worst-case slowdown: fully homomorphic encryption schemes (FHE) [Gen09|BV11a|BV11b|BGV12|Bra12], attribute-based encryption (ABE) schemes [GVW13 $\mathrm{GGH}^{+} 13 \mathrm{~b}$ GGH13a , and functional encryption (FE) schemes for general functions [SS10 GVW12 $\left.\mathrm{GKP}^{+} 13 \mathrm{~b}\right]$.

In this work, we present cryptographic schemes for Turing machines, thus removing the two major limitations of circuits discussed above. We construct attribute-based

\footnotetext{
${ }^{1}$ An exception is the garbling scheme of [LO12] for RAMs, but this scheme also suffers from the worst-case running time problem we address in this paper (see Sec.1.1.
} 
encryption, (succinct and single-key) functional encryption, reusable garbling schemes, and a version of FHE for polynomial-time Turing machines. For each of these schemes, we show that the time to evaluate a Turing machine $M$ on an input $x$ is input specific: it depends on the runtime of $M$ on $x$ and not on the worst-case runtime of $M$ on all inputs of length $n$ where $n=|x|$. Moreover, we show that the token for a Turing machine $M$ is short: its size depends on the size of the description of the Turing machine $M$ and not on $M$ 's runtime. Our schemes are for both uniform and non-uniform Turing machines (so in particular, they can compute circuits).

Our schemes are based on extractable witness encryption, a variant of the witness encryption notion of Garg et al. [GGSW13]. We show how to obtain such an extractable witness encryption scheme using the construction of Garg et al. [GGSW13, by strengthening their assumption with a knowledge property. We prove the new assumption secure in the generic group model. Interestingly, we show that extractable witness encryption is closely related to (weakly) obfuscatable pointfilter functions [GK05].

\subsection{Our Results}

We now explain our results in detail.

Attribute-Based Encryption (ABE) for Turing Machines and RAMs. Attributebased encryption schemes, originally defined by Sahai and Waters [SW05], allow a user holding the master secret key msk to generate a function key $s k_{f}$ for any predicate $f$ of his choice, where $\mathrm{sk}_{f}$ does not hide $f$. Using the master public key mpk, anyone can encrypt a message $m$ with respect to an "attribute" $x$ : such a ciphertext is denoted by $\operatorname{Enc}(x ; m)$. The ciphertext $\operatorname{Enc}(x ; m)$ does not hide $x$, and hides only $m$. Given a function key $\mathrm{sk}_{f}$ and a ciphertext $\operatorname{Enc}(x ; m)$, one can compute $m$ if $f(x)=1$. On the other hand, if $f(x)=0, \mathrm{ABE}$ leaks no information about $m$ and provides semantic security.

Attribute-based encryption is a powerful primitive and has thus received significant attention [GPSW06 $\mathrm{LOS}^{+} 10 \mathrm{LW12GVW13]}$. The state-of-the-art is the scheme of Gorbunov et al. [GVW13]: based on the LWE assumption, they construct an ABE for the class of all circuits of depth at most $d$, where the efficiency of the scheme (such as the size of the ciphertexts) decreases polynomially with $d$. In concurrent work, Garg et al. constructed $\mathrm{ABE}$ schemes with similar properties [GGH$\left.{ }^{+} 13 \mathrm{~b}\right]$, and an $\mathrm{ABE}$ scheme with large ciphertexts [GGSW13], both from candidate multi-linear maps.

In this work, we construct an attribute-based encryption scheme for all circuits, with no restriction on the depth. More importantly, we model functions as Turing machines (with possibly non-uniform advice), as opposed to circuits as in previous work. Computing a function key $\mathrm{sk}_{M}$, corresponding to a Turing machine $M$, takes roughly linear time in the size of the description of $M$, independent of the runtime of $M$. Moreover, given $\mathrm{sk}_{M}$ and $\operatorname{Enc}(x ; m)$ where $f(x)=1$, one can compute $m$ in time that depends only on the time it takes to compute $M$ on input $x$ as opposed to the worst-case running time of $M$. We prove the security of our scheme with respect to a non-adaptive simulation-based definition (we refer the reader to Sec. 3 for details). We then show that a modification of our construction provides ABE for RAMs. 
Theorem 1 (Informal). There exists an attribute-based encryption scheme (as defined in Defs. 3. 4) for (uniform or non-uniform) polynomial-time Turing machines and RAMs from the assumptions in Sec. 1.2

Interestingly, we show how to extend our ABE scheme beyond Turing machines and RAMs: for example, an evaluator can choose by himself which Turing machines to run on the ciphertexts, as long as they satisfy some property expressed in a function key.

Functional Encryption (FE) for Turing Machines. Functional encryption, formalized by Boneh, Sahai and Waters [BSW11], is a generalization of attributebased encryption. In functional encryption, a user holding the master secret key msk can generate a function key $\mathrm{sk}_{f}$ corresponding to a function $f$; then, anyone having a ciphertext $\operatorname{Enc}(x)$ and a function key $\mathrm{sk}_{f}$ can compute $f(x)$, but learns nothing else about the input $x$.

So far, the only many-keys FE schemes known (schemes in which the secret key owner can securely release an unbounded number of function keys) are for the inner-product predicates [KSW08 SSW09]. For general functions, Agrawal et al. AGVW13] showed that there does not exist a many-keys FE scheme if one wants to achieve a natural simulation-based security definition? so the natural question was to construct a single-key functional encryption scheme for general functions. Sahai and Seyalioglu [SS10], Gorbunov et al. [GVW12], and Goldwasser et al. [GKP ${ }^{+} 13 \mathrm{~b}$ ] constructed such schemes for circuits. The work of Goldwasser et al. [GKP $\left.{ }^{+} 13 \mathrm{~b}\right]$ is the first to provide succinct ciphertexts: the ciphertext size is much smaller than the circuit size; they constructed a succinct single-key FE scheme for any depth $d$ circuit, where the parameters of the scheme grow with $d$ (but are independent of the circuit size).

In this work, we not only remove this depth- $d$ restriction, but we model functions as (possibly non-uniform) Turing machines, as opposed to circuits as in prior work. Our schemes have short function keys: computing the function key of a Turing machine $M$ depends only on the size of $M$ and does not depend on the runtime of $M$. We note that in all previous schemes for general functions the size of a function key for a function $f$ grows (at least linearly) with the worst-case runtime of $f$. We note however, that as opposed to our ABE scheme, in a functional encryption scheme, given $\operatorname{Enc}(x)$ and $\mathrm{sk}_{M}$, the time it takes to compute $M(x)$ must be proportional to the worst-case runtime of $M$, since the runtime of $M$ on input $x$ may leak sensitive information about $x$. However, if one is willing to slightly relax security and allow leaking the runtime of $M$ on the secret input $x$, then we provide a second functional encryption scheme for which the decryption algorithm has input-specific runtime (i.e., it runs in time polynomial in the runtime of $M$ on input $x$ ) - we denote this by input-specific runtime functional encryption.

Theorem 2 (Informal). There exists a single-key (succinct) functional encryption scheme and input-specific runtime functional encryption scheme for (uniform or nonuniform) polynomial-time Turing machines from the assumptions in Sec. 1.2

Variant of FHE for Turing Machines. We construct a variant of FHE where one can evaluate a Turing machine $M$ on a ciphertext $\operatorname{Enc}(x)$ in time that depends on the runtime

\footnotetext{
${ }^{2}$ Their lower bound does not apply to weaker security definitions.
} 
of $P$ on the specific input $x$. We naturally call this scheme input-specific FHE. At first glance, this may seem impossible, since revealing the runtime of $P$ on input $x$ may reveal secret information about $x$. However, for many Turing machines $M$, revealing only the runtime of $M$ is not harmful, and it can provide significant efficiency gains.

Our construction is an improvement of Goldwasser et al. [GKP ${ }^{+} 13 \mathrm{~b}$ who showed how to construct input-specific runtime FHE from single-key functional encryption. As in Goldwasser et al. [GKP $\left.{ }^{+} 13 \mathrm{~b}\right]$, we also encrypt a Turing machine $M$ and $x$ together into a token $\mathrm{tk}_{M, x}$. Producing such a token depends only on the size of $x$ and $M$, and not on the running time of $M$. The evaluator can use $\mathrm{tk}_{M, x}$ and public information to compute $M(x)$ in input-specific time. The reason we provide a token for $M$ at all is for security: the FHE evaluator must no longer be able to evaluate TMs of its choice on the encrypted inputs because the running time of those TMs can leak the input entirely. We combine $M$ and $x$ in $\mathrm{tk}_{M, x}$ for a technical reason stemming from the fact that the FE scheme we use in the construction is single-key - we elaborate in our full paper.

Comparing to $\left[\mathrm{GKP}^{+} 13 \mathrm{~b}\right]$, we make the following improvements:

- Remove costly preprocessing. [GKP $\left.{ }^{+} 13 \mathrm{~b}\right]$ had an expensive preprocessing phase taking as long as the worst-case runtime. With our scheme, the preprocessing is cheap: polynomial in the size of the TMs and independent of the worst-case runtime (so in fact it can be performed in the online phase).

- Works for any polynomial-time Turing machine. Because the ciphertext size in $\left[\mathrm{GKP}^{+} 13 \mathrm{~b}\right.$ depended on the depth of the worst-case circuit representation of the class of Turing machines, $\left[\mathrm{GKP}^{+} 13 \mathrm{~b}\right.$ ] only allowed a restricted class of Turing machines: the class of TMs that can be expressed by shallow-depth circuits (e.g., log-space Turing machines). Our result does not have the depth restriction and thus applies to any class of Turing machines with runtime upper-bounded by a polynomial.

Theorem 3 (Informal). There exists an input-specific-runtime fully homomorphic encryption scheme for (uniform or non-uniform) polynomial-time Turing machines based on the assumptions in Sec. 1.2

Reusable Garbling Scheme for Turing Machines. Garbling schemes, introduced in the seminal work of Yao [Yao86], have found many applications in cryptography. In such schemes, a user can "garble" a function $f$ and then encode an input $x$ in a token $\mathrm{tk}_{x}$. Given a garbling of $f$ and a token $\mathrm{tk}_{x}$, one can compute $f(x)$, but learns nothing else about $f$ or $x$. Some works also considered an authenticity property [BHR12 GVW13], on which we do not dwell. Traditional garbling schemes are one-time: they are secure only if an adversary gets a token for at most one input. A reusable garbling scheme is secure when the adversary gets an unbounded number of tokens.

In known garbling schemes (even non-reusable ones), the size of the garbling is as large as the worst-case runtime of $f$. Often, the reason is that programs are modeled as circuits, and the size of the garbling is at least the size of the corresponding circuit. In this work, we construct a (reusable) garbling scheme for (uniform or non-uniform) Turing machines, where the size of the garbling depends only on the size of the Turing machine, and is independent of its runtime. The work of [LO12] is an exception from the circuit model: they model computation as RAM, but their scheme still has large garbling size, at least as large as the worst-case running time. 
As in our FHE and FE schemes, if one allows leaking the runtime of $M$ on input $x$, we can additionally avoid worst-case evaluation time and obtain an input-specific reusable garbling scheme: given a garbling for a Turing machine $M$ and a token $\mathrm{tk}_{x}$, the time to compute $M(x)$ is polynomial in the runtime of $M$ on the specific input $x$.

Goldwasser $e$ al. $\left[\mathrm{GKP}^{+} 13 \mathrm{~b}\right.$ provide a reusable garbling scheme only for depth bounded circuits; our schemes remove the depth dependency, provide short garbling size, and can additionally avoid worst-case running time.

Theorem 4 (Informal). There exists a reusable garbling scheme and an inputspecific reusable garbling scheme for (uniform or non-uniform) polynomial-time Turing machines from the assumptions in Sec. 1.2 .

In summary, our work models computation on encrypted data as Turing machines and thus avoids the worst-case "curse" for a set of well-known cryptographic notions.

Remark 1. Interestingly, we can easily overcome the worst-case curse for interactive tasks such as two-party and multi-party protocols as follows. To securely evaluate a Turing machine $M$, we evaluate the Turing machines $M_{1}, \ldots, M_{\omega(\log n)}$ sequentially, where $M_{i}$ runs the Turing machine $M$ for $2^{i}$ steps and outputs $M$ 's answer if $M$ halted in $2^{i}$ steps, otherwise $\perp$. To evaluate $M_{i}$, we simply use existing multi-party protocols. Note that the circuit size for $M_{i}$ is poly $\left(2^{i}\right)$, and since we halt the computation as soon as we get a non- $\perp$ answer, the protocol runs in input-specific time. The reason we can overcome the worst-case curse in this manner is that interaction is allowed. In this work, we focus on non-interactive tasks, which are more challenging.

\subsection{Our Assumptions}

Our schemes rely on two assumptions: extractable witness encryption and the existence of SNARKs.

Extractable Witness Encryption. The recent work of Garg et al. [GGSW13] constructs a new primitive called witness encryption (WE). Such a scheme is associated with some NP complete language $L$. Given an instance $x$ and a message $m$, any user can encrypt $m$ with respect to $x$; this is denoted by $\operatorname{Enc}_{x}(m)$. Given $\operatorname{Enc}_{x}(m)$ and a valid witness $w$ of $x$, any user can decrypt $x$ efficiently. On the other hand, if $x$ is not in the language, the scheme provides semantic security.

In our work, we additionally assume that the [GGSW13] scheme is extractable: if an adversary can break semantic security for an instance $x$, an extractor can extract the witness for $x$. Such an extractable scheme can be constructed from an extractable version of the [GGSW13] assumption (called extractable DGE No-ExactCover assumption) so we strengthen their assumption. While we state our assumption in a decisional form for simplicity, the search version of the assumption suffices for our schemes because we can use hard-core predicates to mask the one bit we care to hide $(m)$.

We validate our assumption in the generic group model: we prove that no polynomialtime adversary can break the assumption in the generic group model where adversaries can only use multilinear map operations as a black-box. We refer the reader to our full 
paper for more details on the assumption, and emphasize that we view our result as a reduction from any extractable witness encryption scheme, as opposed to a result that is tied to the specific computational assumption.

We show that, interestingly, extractable witness encryption is highly related to another task that was already well-known in the cryptographic literature: (weakly) obfuscating point-filter functions, defined by Goldwasser and Kalai [GK05]. Informally, point-filter functions for a language $L \in \mathbf{N P}$ with witness relation $R_{L}$ are a class of functions $\left\{\delta_{x, b}\right\}$, indexed by a string $x \in\{0,1\}^{n}$ and a bit $b \in\{0,1\}$ that behave as follows:

$$
\delta_{x, b}(w)=\left\{\begin{array}{l}
(x, b), \text { if }(x, w) \in R_{L}, \\
(x, \perp), \text { otherwise. }
\end{array}\right.
$$

It can be shown that extractable witness encryption is indeed equivalent to (weakly) obfuscating point filter function. Thus, the former implies the consequences of the later regarding the impossibility of obfuscation for a wide range of natural tasks based on [GK05]. See our full paper for more details.

The Existence of SNARKs (Succinct Non-Interactive Arguments of Knowledge). Bitansky et al. [BCCT13] construct SNARKs in a generic way (via a reduction from weaker SNARKs). Their work is based on "knowledge of exponent assumptions", and the existence of collision resistant hash functions.

If we remove SNARKs from our constructions, we still obtain novel schemes over prior work because the sizes of the function keys and of the garbling remain short, linear in the size of the Turing machine. Without SNARKs, though, the loss is that the ciphertext size grows with the running time of the Turing machines.

Our FE, FHE, and reusable garbling schemes additionally rely on the existence of a fully homomorphic encryption scheme, which can be obtained from the LWE assumption with circular security [BGV12].

\subsection{Techniques Overview}

ABE for Turing Machines. The main technical challenge in this work is constructing an $\mathrm{ABE}$ scheme for Turing machines.

Our construction starts with witness encryption and a signature scheme. The function key for a Turing machine $M$ is simply a signature of $M$. The master secret and public keys generated during setup are the secret and verification keys (SigSK, VK) for the signature scheme. To encrypt a bit $b$ with respect to a (public) attribute $x$, we compute a witness encryption $\mathrm{Enc}_{x^{*}}(b)$, where $x^{*}=(x, \mathrm{VK})$ and where a valid witness for $x^{*}$ is a tuple $(M, \sigma, \pi)$, where $M$ is a Turing machine, $\sigma$ is a signature of $M$ using SigSK, and $\pi$ the tableau of the computation, which can be interpreted as a "proof" that $M(x)=1$.

Loosely speaking, the security proof proceeds as follows. Suppose there exists a successful adversary $\mathcal{A}$ for our $\mathrm{ABE}$ scheme. Then, given $\mathrm{Enc}_{x^{*}}(b)$, the $\mathrm{ABE}$ encryption of a random bit $b$, and several secret keys $\mathrm{sk}_{M_{i}}=\sigma_{i}$ such that $M_{i}(x)=0$, $\mathcal{A}$ succeeds in guessing $b$ with non-negligible advantage. The security of the extractable witness encryption implies that there exists a poly-time extractor that extracts a valid witness from $\mathcal{A}$ with non-negligible probability. Recall that a valid witness is a triplet of the form $\left(M^{*}, \sigma^{*}, \pi^{*}\right)$ where $\sigma^{*}$ is a valid signature of the Turing machine $M^{*}$ and 
$\pi^{*}$ is a proof that $M^{*}(x)=1$. Note that since $M_{i}(x)=0$ for every $i$, it must be the case that $M^{*} \neq M$, which contradicts the unforgeability of the signature scheme.

Unfortunately, this idea does not quite give us the results we want. The reason is that the time to check a witness for an instance $x^{*}=(x, \mathrm{VK})$ is very long because it involves checking the tableau $\pi$ of $M$ on input $x$. In this case, the witness encryption of Garg et al. [GGSW13] is not "succinct": the size of the ciphertext $\mathrm{Enc}_{x^{*}}(b)$ grows with the time to check the witness. Thus, the approach above gives us a non-succinct ABE scheme, where the size of a ciphertext depends on the worst-case runtime of any (allowed) Turing machine.

To obtain succinctness, we use a SNARG scheme [BCCT13]. A SNARG has a common reference string crs, which is assumed to be securely generated. Any user can prove any NP statement by computing a proof $\pi$. The length of the crs, the length of the proofs, and the time to verify a proof are all short: depending only on the security parameter, and not on the time to verify the NP witness.

$\mathrm{Enc}_{x^{*}}(b)$ now proceeds as follows. It generates a crs corresponding the underlying SNARG scheme. To encrypt a bit $b$ w.r.t. a public attribute $x$, it simply computes Enc $_{x^{*}}(b)$, where $x^{*}$ is now $(x, \mathrm{crs}, \mathrm{VK})$. A valid witness for $x^{*}$ is a tuple of the form $(M, \sigma, \pi)$ where $\sigma$ is a valid signature of the Turing machine $M$, and $\pi$ is a succinct SNARG proof that $M(x)=1$. The fact that $\pi$ can be verified in a short time makes the WE ciphertext succinct, as desired.

This gives us an ABE for Turing machines. Because SNARKs are for NP, our resulting ABE scheme is for any class of Turing machines for which there exists a polynomial that upper bounds the runtime of all machines in the class.

There scheme still has a slight drawback: it is succinct only for uniform Turing machines. If the Turing machines have non-uniform advice as large as the runtime, the resulting ABE ciphertexts are non-succinct. We would like our ABE scheme to be a generalization of previous work on circuits, and in particular to be succinct for any nonuniform Turing machine. To this end, we replace the SNARG scheme with a SNARK scheme (succinct non-interactive argument of knowledge) scheme. SNARKs have the additional property that if an adversary $\mathcal{A}$ succeeds in proving that $x \in L$, an extractor can extract a corresponding witness $w$ from $\mathcal{A}$.

The final $\mathrm{ABE}$ scheme is as before, except that now a valid witness for $x^{*}=$ $(x, \mathrm{crs}, \mathrm{VK})$ is a pair $(\pi, t)$ (without the Turing machine and the signature), where $\pi$ is a proof-of-knowledge of a Turing machine $M$ and a signature $\sigma$ such that $\sigma$ is a valid signature of $M$ and $M(x)=1$. Now the witness size and the verification time is efficient (independent of the size of the Turing machine or its runtime). We refer the reader to Sec. 3 for more details on our ABE scheme and the security proof.

Functional Encryption for Turing Machines. We use the reduction of Goldwasser et al. $\left[\mathrm{GKP}^{+} 13 \mathrm{~b}\right]$ to construct a (single-key and succinct) FE scheme from FHE and ABE. Their reduction is for circuits so we need to adapt it to Turing machines. The main technical issue is that we need to perform the FHE evaluation of a Turing machine $M$. To achieve this goal, we construct a new Turing machine $M_{\mathrm{FHE}}$ that evaluates homomorphically the transition function of $M$ for a $t$ number of times. The problem is that $M_{\mathrm{FHE}}$ needs to know what inputs to read from $M$ 's tape to feed into the FHE evaluation, but the movement of the head in $M$ is an output of the transition function, so 
it is encrypted with FHE and unavailable to $M_{\mathrm{FHE}}$. To solve this issue, we transform $M$ into an oblivious Turing machine using Pippenger-Fischer [PF79]: now the movement of the head follows a fixed and known pattern independent of the input to $M$.

If one allows the runtime of $M$ on $x$ to leak, we can provide a second FE scheme FE $^{*}$ whose decryption algorithm runs in input-specific time. We construct FE* as a reduction from our FE scheme above using the idea of $\left[\mathrm{GKP}^{+} 13 \mathrm{~b}\right]$ : instead of generating a function key $\mathrm{sk}_{M}$ for a Turing machine $M$, we generate many function keys $\mathrm{sk}_{M_{1}}, \ldots, \mathrm{sk}_{M_{\log B_{n}}}$, where $M_{i}$ is the Turing machine that runs $M$ for $2^{i}$ time steps, and either outputs the output of $M$ or $\perp$ if $M$ did not halt in $2^{i}$ steps; the parameter $B_{n}$ is a global bound on the runtime of the Turing machines we consider. To generate $\log B_{n}$ function keys, we use $\log B_{n}$ instances of our single-key functional encryption scheme above, by generating fresh keys for every instance of it. Moreover, since the underlying functional encryption scheme is for Turing machines, generating $\mathrm{sk}_{M_{i}}$ can be done very efficiently, in time polynomial in the size of $M_{i}$, independent on the runtime of $M_{i}$.

On input a ciphertext $\operatorname{Enc}(x)$ and a function key $\left(\mathrm{sk}_{M_{1}}, \ldots, \mathrm{sk}_{M_{\log B}}\right)$ for the Turing machine $M$, the decryption algorithm first tries to decrypt with $\mathrm{sk}_{M_{1}}$, then tries with $\mathrm{sk}_{M_{2}}$, and so on. The first time that it succeeds it stops. Note that the runtime of this decryption algorithm depends on the runtime of $M$ on the specific input $x$, denoted by $t_{x}$. This is the case since it runs the original decryption algorithm (which runs in the worst-case) only with the secret keys $\mathrm{sk}_{M_{1}}, \ldots, \mathrm{sk}_{M_{\log t_{x}}}$, and all the Turing machines $M_{1}, \ldots, M_{\log t_{x}}$ run in time at most $t_{x}$.

Reusable Garbling and a Variant of FHE for Turing Machines. In our full version, we show how to construct these schemes from our FE scheme using a similar reduction to $\left[\mathrm{GKP}^{+} 13 \mathrm{~b}\right]$.

Other Related Work. We discuss other related work in the full version of our paper.

\subsection{Paper Roadmap}

The rest of this paper is organized as follows. We provide definitions for extractable witness encryption and $\mathrm{ABE}$ in Sec. 2 and refer the reader to our full paper [GKP ${ }^{+} 13 \mathrm{a}$ for other relevant preliminaries. Next, Sec. 3 presents our ABE scheme for Turing machines, which we prove formally in our full paper. Finally, Sections 4 and 4.2 show how to construct functional encryption for Turing machines. Due to space constraints, in our full paper $\left[\mathrm{GKP}^{+} 13 \mathrm{a}\right]$, we present the construction of extractable witness encryption and prove the new assumption in the generic group model, we show that extractable witness encryption implies (weakly) obfuscatable point filter functions and deduce implications to obfuscation, and we present the construction of FHE for Turing machines.

\section{Preliminaries}

In this section, we define extractable witness encryption and $\mathrm{ABE}$ for Turing machines, and refer the reader to our full paper for definitions of FE for Turing machines, SNARKs, and other relevant preliminaries. 


\subsection{Notation}

We let $\kappa$ denote the security parameter throughout this paper. For a distribution $\mathcal{D}$, we say $x \leftarrow \mathcal{D}$ when $x$ is sampled from the distribution $\mathcal{D}$. If $S$ is a finite set, by $x \leftarrow S$, we mean $x$ is sampled from the uniform distribution over the set $S$.

We say that a function $f$ is negligible in an input parameter $\kappa$, if for all $d>0$, there exists $K$ such that for all $\kappa>K, f(\kappa)<k^{-d}$. For brevity, we write: for all sufficiently large $\kappa, f(\kappa)=\operatorname{negl}(\kappa)$.

\subsection{Witness Encryption (WE)}

The syntax of WE is as defined by Garg et al. [GGSW13], but the security definition has an additional extractability property.

Definition 1 (Witness Encryption). A witness encryption for a language $L \in N P$ with corresponding witness relation $R_{L}$ consists of two polynomial-time algorithms (WE.Enc, WE.Dec) such that

- Encryption WE.Enc $\left(1^{\kappa}, x, b\right)$ : takes as input a security parameter $\kappa, x \in\{0,1\}^{*}$ and $a$ bit $b$ and outputs a ciphertext $\mathrm{ct}$.

- Decryption WE.Dec $(w, \mathrm{ct})$ : takes as input $w \in\{0,1\}^{*}$ and a ciphertext $\mathrm{ct}$ and outputs a bit b or the symbol $\perp$.

Correctness: For all $(x, w) \in R_{L}$, for all bits $b$, for every sufficiently large security parameter $\kappa$ :

$$
\operatorname{Pr}\left[\mathrm{ct} \leftarrow \mathrm{WE} \cdot \operatorname{Enc}\left(1^{\kappa}, x, b\right): \operatorname{WE} \cdot \operatorname{Dec}(w, \mathrm{ct})=b\right]=1-\operatorname{negl}(\kappa) .
$$

Definition 2 (Extractable security). A witness encryption scheme for a language $L \in$ $N P$ is secure if for all p.p.t. adversaries $A$, and all poly q, there exists a p.p.t. extractor $E$ and a poly $p$, such that for all auxiliary inputs $z$ and for all $x \in\{0,1\}^{*}$, the following holds:

$$
\begin{aligned}
\operatorname{Pr}[b \leftarrow\{0,1\} ; \mathrm{ct} \leftarrow & \text { WE.Enc } \left.\left(1^{\kappa}, x, b\right): A(x, \mathrm{ct}, z)=b\right] \geq 1 / 2+1 / q(|x|) \\
& \Rightarrow \operatorname{Pr}\left[E(x, z)=w:(x, w) \in R_{L}\right] \geq 1 / p(|x|) .
\end{aligned}
$$

\subsection{Attribute-Based Encryption (ABE) for Turing Machines}

We define the syntax and security of $\mathrm{ABE}$ for Turing machines.

Definition 3 (ABE for Turing machines). An attribute-based encryption scheme $\mathrm{ABE}$ for a class of Turing machines $\mathcal{T}$ is a tuple of four algorithms (ABE.Setup, ABE.KeyGen, ABE.Enc, ABE.Dec), the first three of which are p.p.t., such that:

- ABE.Setup $\left(1^{\kappa}\right)$ takes as input the security parameter $1^{\kappa}$ and outputs a master public key mpk and a master secret key msk.

- ABE.KeyGen(msk, $M)$ takes as input the master secret key msk, a Turing machine $M \in \mathcal{T}$, and outputs a function key $\mathrm{sk}_{M}$. 
- ABE.Enc $(\mathrm{mpk}, x, b)$ takes as input the master public key mpk, an attribute $x \in$ $\{0,1\}^{*}$, and $a$ bit $b$ and outputs a ciphertext $\mathrm{ct}$.

- ABE.Dec $\left(\mathrm{sk}_{M}, \mathrm{ct}\right)$ takes as input a key $\mathrm{sk}_{M}$ and a ciphertext c and outputs a bit.

Correctness. For all Turing machines $M \in \mathcal{T}$, for all attributes $x \in\{0,1\}^{*}$, for all bits $b$, for $\kappa$ sufficiently large,

$$
\begin{aligned}
& \operatorname{Pr}\left[(\mathrm{mpk}, \mathrm{msk}) \leftarrow \operatorname{ABE} . \operatorname{Setup}\left(1^{\kappa}\right) ; \mathrm{fsk}_{f} \leftarrow \mathrm{ABE} . \text { KeyGen }(\mathrm{fmsk}, f)\right. \\
& \left.\quad c \leftarrow \operatorname{ABE} . \operatorname{Enc}(\mathrm{fmpk}, x): \mathrm{ABE} . \operatorname{Dec}\left(\mathrm{fsk}_{f}, 1^{t}, c\right)=f(x)\right] \\
& \quad=1-\operatorname{negl}(\kappa)
\end{aligned}
$$

Efficiency. There exists a polynomial p such that the running time of $\mathrm{ABE} . \operatorname{Dec}\left(\mathrm{sk}_{M}, \mathrm{ct}\right)$ is at most $p(\kappa$, runtime $(M, x))$.

The efficiency property states that the work of the decryption depends on the run time of a Turing machine on the attribute. Since ABE.Setup, ABE.KeyGen and ABE.Enc are p.p.t.-s, their running time depends only on the security parameter and not on the running time of the Turing machines (except for a logarithmic dependency on it).

Our security definition is full (the adversary can choose the challenge attribute based on the public key) and non-adaptive (the adversary chooses the Turing machines before getting the challenge ciphertext).

Definition 4 (Attribute-based encryption security). Let ABE be an attribute-based encryption scheme for a class of Turing machines $\mathcal{T}$ and let $A=\left(A_{1}, A_{2}\right)$ be an adversary. Consider the following experiment.

\section{$\underline{\operatorname{Exp}_{\mathrm{ABE}}\left(1^{\kappa}\right):}$}

1: $($ mpk, msk $) \leftarrow$ ABE.Setup $\left(1^{\kappa}\right)$

2: $(x$, state $) \leftarrow A_{1}^{\mathrm{ABE} . \mathrm{KeyGen}(\mathrm{msk}, \cdot)}(\mathrm{mpk})$

3: Choose a bit $b$ at random and let ct $\leftarrow$ ABE.Enc $(\mathrm{mpk}, x, b)$.

4: $b^{\prime} \leftarrow A_{2}$ (state, ct).

5: If, $b=b^{\prime}$ and for all Turing machines $M$ that $A$ requests to oracle ABE.KeyGen $(\mathrm{msk}, \cdot)$, we have $M(x)=0$, output 1 , else output 0 .

We say that the scheme is a secure attribute-based encryption for Turing machines if for all p.p.t. adversaries $A$, and for all sufficiently large $\kappa$ :

$$
\operatorname{Adv}_{\mathrm{ABE}, A}:=\left|\operatorname{Pr}\left[\operatorname{Exp}_{\mathrm{ABE}, A}\left(1^{\kappa}\right)=1\right]-1 / 2\right|=\operatorname{negl}(\kappa) .
$$

\section{Attribute-Based Encryption for Turing Machines and RAMs}

We construct an $\mathrm{ABE}$ scheme for Turing machines based on three ingredients:

1. an extractable witness encryption scheme WE = (WE.Enc, WE.Dec) based on the work of [GGSW13], on which we elaborate in Sec.2.2, 
2. a succinct argument of knowledge scheme, SNARK = (SNARK.Gen, SNARK.Prover, SNARK.Verify), based on the work of [BCCT13],

3. an existentially unforgeable signature scheme secure against adaptive chosen message attacks SIG = (SIG.KeyGen,SIG.Sign, SIG.Verify) [GMR88].

Theorem 5. Assuming the above three primitives, there exists a secure attribute-based encryption scheme (as per Def. 4) for any class of (uniform or non-uniform) Turing machines $\mathcal{T}$, for which there exists a polynomial $p$ such that the runtime of every machine in $\mathcal{T}$ is upper-bounded by $p$.

The $p$ restriction comes from the fact that SNARKs are for NP. From now on, for brevity, we will refer to such a class by "a class of Turing machines with runtime upperbounded by some polynomial".

Corollary 1. There exists a secure attribute-based encryption scheme for any class of (uniform or non-uniform) Turing machines whose runtime is upper-bounded by some polynomial under the extractable DGE No-Exact-Cover assumption, "knowledge of exponent assumption", and the existence of collision-resistant hash functions (Sec. 1.2).

\subsection{Construction preliminaries}

We advise the reader to recall the intuition we provided in technique overview, Sec. 1.3 The Language $L$ for SNARK. We define $L$ by defining its relation, $R_{L}$. Let $R_{L}$ be the following instance-witness relation: the instance is of the form $y=(\mathrm{VK}, x, t)$ (a verification key $\mathrm{VK}$ for a signature scheme, an input $x$, and a time bound $t$ ) and the witness is of the form $w=(M, \sigma)$, for $M$ a Turing machine and $\sigma$ a signature. Then, $(y, w) \in R_{L}$ iff SIG.Verify $(\mathrm{VK}, M, \sigma)=1$ and $M$ halts on $x$ in at most $t$ steps and outputs one. Moreover, $t<p(|x|)$, where $p$ is a polynomial upper-bound on the runtime of every Turing machine in the class of interest. Let (SNARK.Gen, SNARK.Prover, SNARK.Verify) be a SNARK system for $L$.

The Language $L^{*}$ for WE. Based on the above language $L$ and the SNARK system (SNARK.Gen, SNARK.Prover, SNARK.Verify) for $L$, we define a language $L^{*}$ for the witness encryption scheme using the witness relation $R_{L^{*}}$ as follows:

$$
R_{L^{*}}\left[x^{*}=(x, \mathrm{crs}, \mathrm{VK}), w^{*}=(\pi, t)\right]=1 \text { iff SNARK.Verify }(\mathrm{crs},(\mathrm{VK}, x, t), \pi)=1
$$

Let $W E=($ WE.Enc, WE.Dec) be an extractable witness encryption scheme for the witness relation $R_{L^{*}}$.

\subsection{Construction of ABE for Turing Machines}

Our construction of $A B E=(A B E . S e t u p, A B E . K e y G e n, A B E . E n c, A B E . D e c)$ for Turing machines proceeds as follows. Let $\mathcal{T}$ be the class of (uniform or non-uniform) polynomial time Turing machines for the ABE scheme.

Setup ABE.Setup $\left(1^{\kappa}\right)$ where $\kappa$ is the security parameter: 
1. Sample a verification key / signing key pair $($ VK, SigSK $) \leftarrow \operatorname{SIG.KeyGen}\left(1^{\kappa}\right)$, and output $\mathrm{mpk}:=\mathrm{VK}$ and $\mathrm{msk}:=$ SigSK.

Encryption ABE.Enc(mpk, $x, b)$ where mpk $=\mathrm{VK}, x \in\{0,1\}^{*}$ and $b \in\{0,1\}$ :

1. Run the SNARK generator SNARK.Gen to get crs $\leftarrow \operatorname{SNARK.Gen}\left(1^{\kappa}\right)$.

2. Let $x^{*}=(x, \mathrm{crs}, \mathrm{VK})$. Compute ctwE $\leftarrow$ WE. Enc $\left(1^{\kappa}, x^{*}, b\right)$.

3. Output $\mathrm{ct}:=\left(x^{*}, \mathrm{ct}_{\mathrm{WE}}\right)$.

Key generation ABE.KeyGen(msk, $M)$ where $M$ is a Turing machine:

1. Compute $\sigma \leftarrow$ SIG.Sign(SigSK, $M)$ and output sk $\mathrm{sk}_{M}:=(M, \sigma)$.

Decryption $\mathrm{ABE} . \operatorname{Dec}\left(\mathrm{sk}_{M}, \mathrm{ct}\right)$ where $\mathrm{sk}_{M}=(M, \sigma)$ and $\mathrm{ct}=\left(x^{*}=(x, \mathrm{crs}, \mathrm{VK})\right.$, (tWE):

1. Run $M$ on $x$ and let $t$ be the number of steps after which $M$ halts (note that $M$ is a polynomial time Turing machine so it must halt within a polynomial number of steps).

2. If $M(x)=0$, output $\perp$ and exit.

3. Otherwise, let $w:=(M, \sigma)$ and note that $((\mathrm{VK}, x, t), w) \in R_{L}$.

4. Run SNARK.Prover to obtain a proof $\pi \leftarrow$ SNARK.Prover $(\operatorname{crs},(\mathrm{VK}, x, t), w)$.

5. Let $w^{*}=(\pi, t)$. Compute and output WE.Dec $\left(w^{*}, \operatorname{ct}_{\mathrm{WE}}\right)$.

Proof Intuition. We prove Th. 5 formally in our full version, and we only provide intuition here for the security proof. We start by assuming the ABE scheme is not secure, and reach a contradiction by showing that one can forge signatures using the extractability properties of the WE and SNARK schemes. Therefore, assume there is an adversary for $\mathrm{ABE}, A_{\mathrm{ABE}}=\left(A_{\mathrm{ABE}, 1}, A_{\mathrm{ABE}, 2}\right)$. We will show how to construct an adversary $A_{\mathrm{WE}}$ for the WE scheme: $A_{\mathrm{WE}}$ simply embeds its challenge ciphertext into the ciphertext for $A_{\mathrm{ABE}}$ and lets $A_{\mathrm{ABE}}$ decide.

Once we have the adversary $A_{\mathrm{WE}}$, by the security definition of WE, we also have an extractor $E_{\mathrm{WE}}$ which on input $x^{*}$, outputs a valid witness $w^{*}=(\pi, t)$ of $\left(x^{*}, w^{*}\right) \in$ $R_{L^{*}}$. Using $E_{\mathrm{WE}}$, we construct a prover $P^{*}$ for the SNARK system that is able to construct an instance $y=(\mathrm{VK}, x, t)$ and a proof $\pi$ for which the SNARK verifier accepts. By the proof of knowledge property of the SNARK, there exists an extractor $E_{\text {SNARK }}$ that outputs a witness for the SNARK language $L$, namely $w=(M, \sigma)$, such that $(y, w) \in R_{L}$. This means that $M(x)=1$ and that $\sigma$ is a correct signature on $M$; but $A_{\mathrm{ABE}}$ only asked for signatures of Turing machines $M_{i}$ for which $M_{i}(x)=0$. Therefore, $(M, \sigma)$ are a new signature pair and thus we used $P^{*}$ and $E_{\text {SNARK }}$ to forge a signature and reach a contradiction.

\subsection{ABE for RAMs}

In this section, we discuss how to construct ABE for RAMs. This construction is similar to our construction for Turing machines, so we only mention the main differences here: the language $L$ for the SNARK and ABE.KeyGen. See our full paper for more details. Let $(M, D)$ be a RAM pair: a RAM machine $M$ and memory $D$. 
The Language $L$ for SNARK. Let $R_{L}$ be the following instance-witness relation: the instance is of the form $y=(\mathrm{VK}, x, t)$ (a verification key $\mathrm{VK}$ for a signature scheme, an input $x$, and a time bound $t$ ) and the witness is of the form $w=$ $\left(r, M, \sigma_{(r, M)}, S,\left\{i, D_{i}, \sigma_{\left(r, i, D_{i}\right)}\right\}_{i \in S}\right)$, where $r$ is a nonce, $M$ a machine, $\sigma_{(M, r)}$ is a signature on the description of the machine $M$ and the nonce $r, S$ is a set of integers that represent memory addresses (the memory accesses $M$ makes to $D$ ), $D_{i}$ is the value in the $i$-th slot of memory and $\sigma_{r, i, D_{i}}$ is a signature on $r$ and $D_{i}$. Then, $(y, w) \in R_{L}$ iff

1. SIG.Verify $\left(\mathrm{VK},(r, M), \sigma_{(r, M)}\right)=1$,

2. SIG.Verify $\left(\mathrm{VK},\left(r, i, D_{i}\right), \sigma_{\left(r, i, D_{i}\right)}\right)=1$ for all $i \in S$,

3. $M$ halts on $x$ in at most $t$, all of its memory queries are in $S$, and outputs one.

Key generation ABE.KeyGen(msk, $M, D)$ where $M$ is a RAM and $D$ its memory:

1. Choose $r \leftarrow\{0,1\}^{\text {poly }(\kappa)}$.

2. Compute $\sigma_{(r, M)} \leftarrow \operatorname{SIG} \cdot \operatorname{Sign}(\operatorname{SigSK},(r, M))$.

3. For every $i \in 1 \ldots|D|$, compute $\sigma_{\left(r, i, D_{i}\right)} \leftarrow \operatorname{SIG.Sign}\left(\operatorname{SigSK},\left(r, i, D_{i}\right)\right)$.

4. Output $\left(r, M, \sigma_{(r, M)},\left\{D_{i}, \sigma_{\left(r, i, D_{i}\right)}\right\}_{i=1}^{|D|}\right)$.

Key generation runtime and the function key size are polynomial in the description of the RAM and the size of $|D|$, but they do not depend on the runtime of the RAM. (As a remark, to obtain a slightly shorter key size, one can sign a Merkle tree over the entries in $D$.) The time to decrypt also only depends on the time to run the RAM and not on its worst case running time or on the memory size.

\subsection{Beyond ABE for Turing Machines and RAMs}

Interestingly, it turns out the expressivity of our $\mathrm{ABE}$ construction goes beyond that of Turing machines and RAMs. The ABE construction can be easily changed to allow the evaluator to provide an additional input $\alpha$ to the computation. That is, given a function key $\mathrm{sk}_{M}$, a ciphertext $\mathrm{ct}_{x, m}$, an evaluator can choose an input $\alpha$ by himself; then if $M(x, \alpha)=1$, ABE.Dec outputs $m$, otherwise, it outputs $\perp$. To construct such an ABE, one only has to change the SNARK language $L$ such that an instance has the form (VK, $x, t)$ and a witness is $(M, \sigma, \alpha)$ with $M(x, \alpha)=1$ and $\sigma$ verifies $M$.

This extra input $\alpha$ makes the scheme significantly more expressive. We illustrate on two examples. The first example allows the secret key owner to delegate the choice of Turing machines to another user, say Alice, by issuing a function key for Alice; then Alice can choose Turing machines of her choice to run on the ciphertexts, without contacting the secret key owner. To construct this example, the secret key owner generates $s k_{U_{\text {Alice }}}$ where $U_{\text {Alice }}$ is a universal circuit containing Alice's public key. $U_{\text {Alice }}$ takes as input $\alpha=(\mathrm{TM}, \sigma(\mathrm{TM}))$ and $x$ : it first checks that $\sigma(\mathrm{TM})$ verifies with Alice's public key as being a signature of TM, and if so, it runs TM $(x)$. Now Alice can choose any Turing machine TM she wishes, and as long as she signs it, she will be able to evaluate it on the ciphertext. In fact, the secret key owner can delegate the choice of Turing machines to any group of people, and he can even express complex policies, e.g. "allow any Turing machine that is signed by (Alice and Bob) or Chris".

The second example is to run any approved RAM on any approved database, where approved means that it was signed by the secret key owner. We do not elaborate further on this construction and its applications in this short paper version. 


\section{Functional Encryption for Turing Machines}

In this section we construct a (single-key and succinct) functional encryption scheme for Turing machines. We refer the reader to our full paper for a definition of FE for Turing machines.

Theorem 6. Assuming we have:

- an attribute-based encryption scheme for any class of (uniform or non-uniform) Turing machines with running time upper-bounded by a polynomial, and

- a fully homomorphic encryption scheme,

there is a (single-key and succinct) functional encryption scheme for any class of (uniform or non-uniform) Turing machines with running time upper-bounded by a polynomial.

Theorem 7. Assuming there exists a (single-key and succinct) functional encryption scheme for any class of (uniform or non-uniform) Turing machines with running time bounded by a polynomial, there is a (single-key and succinct) input-specific runtime functional encryption scheme for any class of (uniform or non-uniform) Turing machines with running time bounded by a polynomial.

Corollary 2. There exists a secure (single-key and succinct) functional encryption scheme FE and a (single-key) input-specific runtime functional encryption scheme FE* for any class of (uniform or non-uniform) Turing machines with runtime bounded by a polynomial under the extractable DGE No-Exact-Cover assumption, "knowledge of exponent assumption”, and the LWE assumption with circular security (Sec.1.2).

\subsection{FE for Turing Machines Construction (FE)}

Recall the construction overview provided in Sec. 1.3. We follow the reduction of Goldwasser et al. $\left[\mathrm{GKP}^{+} 13 \mathrm{~b}\right]$ who showed how to construct a (single-key and succinct) functional encryption scheme from any ABE and FHE scheme, where functions were modeled as circuits.

Our construction of FE = (FE.Setup, FE.KeyGen, FE.Enc, FE.Dec) proceeds similarly to the $\left[\mathrm{GKP}^{+} 13 \mathrm{~b}\right]$ construction, with the main difference being that we work with Turing machines instead of circuits. There are two places in the reduction where the treatment of circuits is different from the treatment of Turing machines: in the use of the ABE and FHE schemes. To adapt the reduction to Turing machines, we first use our ABE for Turing machines scheme. Second, we need to construct a Turing machine $M_{\mathrm{FHE}}$ that performs the FHE evaluation of another Turing machine $M$. We only present here the construction of $M_{\mathrm{FHE}}$ and delegate the full FE construction to our full paper.

Based on the intuition provided in Sec.1.3, we describe a compiler Compile $\mathrm{FHE}_{\mathrm{FH}}$ that takes as input a Turing machine $M$ and a number of steps $t$ and produces a Turing machine $M_{\mathrm{FHE}}$ that computes the FHE evaluation of $M$ for $t$ steps. In the following, let $\hat{x}$ denote the FHE encryption of $x$. 


\section{Algorithm 1. (Compile $\mathrm{FHE}_{\mathrm{FH}}(M, t)$ )}

1. Use the Pippenger-Fischer transformation [PF79] for time bound $t$ to transform $M$ into an oblivious Turing machine $M_{O}$ with head movement function next. next is a function that takes as input $i$, the current step in the computation, and outputs whether the head of $M_{O}$ should move left or right on the tape. The Turing machine $M_{O}$ has a transition function $\delta: \delta$ takes as input a tape input bit $b$, a state state and outputs a new state state ${ }^{\prime}$, and the new content $b^{\prime}$ for the new tape location which is indicated by next.

2. Based on ( $M_{O}$, next), construct a new Turing machine $M_{\mathrm{FHE}}$ that takes as input an FHE public key hpk and an input encryption $\hat{x}$. $M_{\mathrm{FHE}}$ evaluates homomorphically the transition function $\delta$ of $M_{O}$ for $t$ steps. Each cell of the tape of $M_{O}$ corresponds to the FHE encryption of the cell value for $M_{\mathrm{FHE}}$. At step $i, M_{\mathrm{FHE}}$ maintains the FHE encryption of the state of $M_{O}$ at time $i$ : $\widehat{\text { state }_{i}}$. At step $i, M_{\mathrm{FHE}}$ takes as input the encrypted bit from the input tape $\hat{b}$ that the head currently points at, the current encrypted state $\widehat{\text { state }_{i}}$, and outputs an encrypted new state state $\mathrm{str}_{i+1}$ and a new content $\widehat{b^{\prime}} . M_{\mathrm{FHE}}$ updates the current cell with $\widehat{b^{\prime}}$ and then computes next $(i)$ to determine whether to move left or right.

3. Output the description of $M_{\mathrm{FHE}}$.

Note that the running time of Compile $\mathrm{FHE}_{\text {and }} M_{\mathrm{FHE}}$ is polynomial in $t$.

\subsection{Input-Specific Runtime Functional Encryption for Turing Machines (FE*)}

In what follows we show how to convert a (single-key) functional encryption scheme for Turing machines FE into one where the decryption algorithm, on input a function key for $M$ denoted $\mathrm{fsk}_{M}$ and FE.Enc(MPK, $x$ ), runs in time that depends on the runtime of $M$ on input $x$. Denote by $\mathrm{FE}^{*}$ such a functional encryption scheme. We refer the reader to Sec. 1.3 for the construction overview and to our full paper for the definition of input-specific runtime functional encryption.

Setup FE* $\cdot \operatorname{Setup}\left(1^{\kappa}\right)$ :

1. Generate $\tau:=\log B_{n}$ independent pair of keys for the FE scheme: $\left(\mathrm{msk}_{i}, \mathrm{mpk}_{i}\right) \leftarrow$ FE.Setup $\left(1^{\kappa}\right)$.

2. Output MPK :=(mpk $\left.1, \ldots, \mathrm{mpk}_{\tau}\right)$ and MSK $:=\left(\mathrm{msk}_{1}, \ldots, \mathrm{msk}_{\tau}\right)$.

Key Generation FE* .KeyGen $(\mathrm{MSK}, M)$ : with $\mathrm{MSK}=\left(\mathrm{msk}_{1}, \ldots, \mathrm{msk}_{\tau}\right)$.

1. Let $M_{i}$ be the Turing machine that runs $M$ for $2^{i}$ steps and outputs $M(x)$ if $M$ finishes in that number of steps, otherwise, it outputs $\perp$. Let $t_{i}$ be the number of steps $M_{i}$ runs for 3

2. Let $\mathrm{fsk}_{M_{i}} \leftarrow$ FE.KeyGen $\left(\mathrm{msk}_{i}, M_{i}, t_{i}\right)$, for $i=1 \ldots \tau$.

3. Output $\mathrm{fsk}_{M}:=\left(\mathrm{fsk}_{M_{1}}, \ldots, \mathrm{fsk}_{M_{\tau}}\right)$.

Encryption FE*.Enc $(M P K, x)$ with $\mathrm{MPK}=\left(\mathrm{mpk}_{1}, \ldots, \mathrm{mpk}_{\tau}\right)$

1. Compute $\mathrm{ct}_{i} \leftarrow \mathrm{FE}$. Enc $\left(\mathrm{mpk}_{i}, x\right)$ for $i=1 \ldots \tau$.

\footnotetext{
${ }^{3}$ Note that $t_{i}$ may be slightly larger than $2^{i}$, since $t_{i}$ is the number of steps it takes to simulate a Turing machine that runs for $2^{i}$ steps.
} 
2. Output $\mathrm{ct}:=\left(\mathrm{ct}_{1}, \ldots, \mathrm{ct}_{\tau}\right)$.

Decryption FE* ${ }^{*} \operatorname{Dec}\left(\mathrm{fsk}_{M}, \mathrm{ct}\right)$ : for $\mathrm{fsk}_{M}=\left(\mathrm{fsk}_{M_{1}}, \ldots, \mathrm{fsk}_{M_{\tau}}\right), \mathrm{ct}=\left(\mathrm{ct}_{1}, \ldots, \mathrm{ct}_{\tau}\right)$.

1. Starting with $i=1$, repeat until $v \neq \perp$ :

(a) $v \leftarrow$ FE.Dec $\left(\mathrm{fsk}_{M_{i}}, \mathrm{ct}_{i}\right)$

(b) $i \leftarrow i+1$

2. Output $v$.

Based on this construction, we prove Th.77in our full paper.

Acknowledgments. We would like to thank the authors of the witness encryption paper [GGH13a] and Zvika Brakerski for useful discussions. This work was supported by an NSERC Discovery Grant, by DARPA awards FA8750-11-2-0225 and N6600110-2-4089, by NSF awards CNS-1053143 and IIS-1065219, and by Google. The U.S. Government is authorized to reproduce and distribute reprints for governmental purposes notwithstanding any copyright notation thereon. The views and conclusions contained herein are those of the author and should not be interpreted as necessarily representing the official policies or endorsements, either expressed or implied, of DARPA or the U.S. Government.

\section{References}

AGVW13. Agrawal, S., Gorbunov, S., Vaikuntanathan, V., Wee, H.: Functional encryption: New perspectives and lower bounds. In: Canetti, R., Garay, J.A. (eds.) CRYPTO 2013, Part II. LNCS, vol. 8043, pp. 500-518. Springer, Heidelberg (2013)

BCCT13. Bitansky, N., Canetti, R., Chiesa, A., Tromer, E.: Recursive composition and bootstrapping for SNARKs and proof-carrying data. In: STOC (2013)

BGV12. Brakerski, Z., Gentry, C., Vaikuntanathan, V.: (Leveled) fully homomorphic encryption without bootstrapping. In: ITCS, pp. 309-325 (2012)

BGW88. Ben-Or, M., Goldwasser, S., Wigderson, A.: Completeness theorems for noncryptographic fault-tolerant distributed computation. In: STOC, pp. 1-10 (1988)

BHR12. Bellare, M., Hoang, V.T., Rogaway, P.: Foundations of garbled circuits. In: ACM CCS, pp. 784-796 (2012)

Bra12. Brakerski, Z.: Fully homomorphic encryption without modulus switching from classical gapSVP. In: Safavi-Naini, R., Canetti, R. (eds.) CRYPTO 2012. LNCS, vol. 7417, pp. 868-886. Springer, Heidelberg (2012)

BSW11. Boneh, D., Sahai, A., Waters, B.: Functional encryption: Definitions and challenges. In: Ishai, Y. (ed.) TCC 2011. LNCS, vol. 6597, pp. 253-273. Springer, Heidelberg (2011)

BV11a. Brakerski, Z., Vaikuntanathan, V.: Efficient fully homomorphic encryption from (standard) LWE. In: FOCS, pp. 97-106 (2011)

BV11b. Brakerski, Z., Vaikuntanathan, V.: Fully homomorphic encryption from ring-LWE and security for key dependent messages. In: Rogaway, P. (ed.) CRYPTO 2011. LNCS, vol. 6841, pp. 505-524. Springer, Heidelberg (2011)

CCD88. Chaum, D., Crépeau, C., Damgård, I.: Multiparty unconditionally secure protocols (extended abstract). In: STOC, pp. 11-19 (1988) 
Gen09. Gentry, C.: Fully homomorphic encryption using ideal lattices. In: STOC, pp. 169-178 (2009)

GGH13a. Garg, S., Gentry, C., Halevi, S.: Candidate multilinear maps from ideal lattices. In: Johansson, T., Nguyen, P.Q. (eds.) EUROCRYPT 2013. LNCS, vol. 7881, pp. 1-17. Springer, Heidelberg (2013)

GGH $^{+}$13b. Garg, S., Gentry, C., Halevi, S., Sahai, A., Waters, B.: Attribute-based encryption for circuits from multilinear maps (2013)

GGSW13. Garg, S., Gentry, C., Sahai, A., Waters, B.: Witness encryption and its applications. In: STOC (2013)

GK05. Goldwasser, S., Kalai, Y.T.: On the impossibility of obfuscation with auxiliary input. In: FOCS, pp. 553-562 (2005)

$\mathrm{GKP}^{+}$13a. Goldwasser, S., Kalai, Y.T., Popa, R.A., Vaikuntanathan, V., Zeldovich, N.: How to run Turing machines on encrypted data. Cryptology ePrint Archive, Report 2013/229 (2013), http://eprint.iacr.org/

GKP $^{+}$13b. Goldwasser, S., Kalai, Y.T., Popa, R.A., Vaikuntanathan, V., Zeldovich, N.: Reusable garbled circuits and succinct functional encryption. In: STOC (2013)

GMR88. Goldwasser, S., Micali, S., Rivest, R.L.: A digital signature scheme secure against adaptive chosen-message attacks. SIAM J. Comput., 281-308 (1988)

GMW87. Goldreich, O., Micali, S., Wigderson, A.: How to play any mental game. In: STOC, pp. 218-229 (1987)

GPSW06. Goyal, V., Pandey, O., Sahai, A., Waters, B.: Attribute-based encryption for finegrained access control of encrypted data. In: ACM CCS, pp. 89-98 (2006)

GVW12. Gorbunov, S., Vaikuntanathan, V., Wee, H.: Functional encryption with bounded collusions via multi-party computation. In: Safavi-Naini, R., Canetti, R. (eds.) CRYPTO 2012. LNCS, vol. 7417, pp. 162-179. Springer, Heidelberg (2012)

GVW13. Gorbunov, S., Vaikuntanathan, V., Wee, H.: Attribute-based encryption for circuits. In: STOC (2013)

KSW08. Katz, J., Sahai, A., Waters, B.: Predicate encryption supporting disjunctions, polynomial equations, and inner products. In: Smart, N.P. (ed.) EUROCRYPT 2008. LNCS, vol. 4965, pp. 146-162. Springer, Heidelberg (2008)

LO12. Lu, S., Ostrovsky, R.: How to garble RAM programs? In: Johansson, T., Nguyen, P.Q. (eds.) EUROCRYPT 2013. LNCS, vol. 7881, pp. 719-734. Springer, Heidelberg (2013)

$\operatorname{LOS}^{+}$10. Lewko, A., Okamoto, T., Sahai, A., Takashima, K., Waters, B.: Fully secure functional encryption: Attribute-based encryption and (Hierarchical) inner product encryption. In: Gilbert, H. (ed.) EUROCRYPT 2010. LNCS, vol. 6110, pp. 62-91. Springer, Heidelberg (2010)

LW12. Lewko, A., Waters, B.: New proof methods for attribute-based encryption: Achieving full security through selective techniques. In: Safavi-Naini, R., Canetti, R. (eds.) CRYPTO 2012. LNCS, vol. 7417, pp. 180-198. Springer, Heidelberg (2012)

PF79. Pippenger, N., Fischer, M.J.: Relations among complexity measures. J. ACM 26(2), 361-381 (1979)

SS10. Sahai, A., Seyalioglu, H.: Worry-free encryption: functional encryption with public keys. In: ACM CCS, pp. 463-472 (2010)

SSW09. Shen, E., Shi, E., Waters, B.: Predicate privacy in encryption systems. In: Reingold, O. (ed.) TCC 2009. LNCS, vol. 5444, pp. 457-473. Springer, Heidelberg (2009)

SW05. Sahai, A., Waters, B.: Fuzzy identity-based encryption. In: Cramer, R. (ed.) EUROCRYPT 2005. LNCS, vol. 3494, pp. 457-473. Springer, Heidelberg (2005)

Yao86. Yao, A.C.: How to generate and exchange secrets (extended abstract). In: FOCS, pp. 162-167 (1986) 